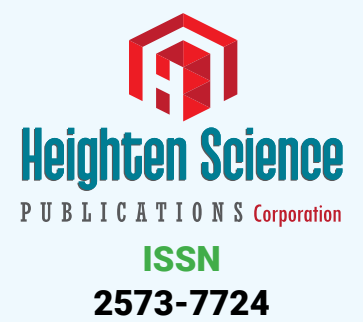

*Address for Correspondence: Austin Fletcher, Saint James School of Medicine, the Quarter, Anguilla, Tel: 872-216-7179; Email: mr.austinfletcher@gmail.com

Submitted: 13 July 2017

Approved: 26 July 2017

Published: 27 July 2017

Copyright: @2 2017 Fletcher A, et al. This is an open access article distributed under the Creative Commons Attribution License, which permits unrestricted use, distribution, and reproduction in any medium, provided the original work is properly cited.

Keywords: Chondromyxoid fibroma; Benign; Curettage; Bone graft; Autologous
Case Report

\section{Radiological evaluation of a Chondromyxoid Fibroma}

\author{
Austin Fletcher* and Megan Mastragostino \\ Saint James School of Medicine, the Quarter, Anguilla
}

\section{ABSTRACT}

Chondromyxoid fibroma (CMF) is a very rare benign cartilaginous tumor representing less than $0.5 \%$ of all bone tumors while also being the rarest cartilaginous bone tumor. Common locations of occurrence include the metaphysial region of the proximal tibia and distal femur. We report a case of a 10-year-old female affected by a CMF of the left lower tibia. The radiological features demonstrated by X-ray and magnetic resonance imaging (MRI) are discussed.

\section{INTRODUCTION}

Chondromyxoid fibroma is a very rare benign cartilaginous tumor representing less than $0.5 \%$ of all bone tumors while also being the rarest cartilaginous bone tumor [1]. The most commonly affected area is the metaphysial region of the proximal tibia and the distal femur [2]. Rarer locations include the fibula, scapula, vertebrae and ribs [3]. The tumor primarily has a male sex distribution, usually presenting before age 30 [2]. We present a case of a CMF occurring in the distal tibia, differing from the most common presentation of the proximal tibia.

\section{CASE REPORT}

A 10-year-old female presented with left lower leg pain that had progressively increased over the duration of 1 year. On physical examination, tenderness was elicited on palpation of the distal tibia. Routine laboratory testing was normal. X-Ray was performed and revealed a lytic lesion with sclerotic margins in the left lower tibia (Figure 1). MRI was subsequently performed, demonstrating a left lower tibia lesion with low signal intensity on T1 weighted images (Figure 2), and high signal intensity on T2 weighted images (Figure 3). Pathology of the tumor revealed a diagnosis of a CMF. She was treated with curettage and autologous bone grafting (Figure 4). Post op eratively the left leg was immobilized with a plaster cast with restricted weight bearing (Figure 5). Follow up X-ray after 5 years demonstrates no tumor reoccurrence (Figure 6, Figure 7).

\section{DISCUSSION}

CMF is the rarest benign cartilaginous bone tumor [4]. Although benign the tumor is locally aggressive in nature [2]. Malignant transformation is very rare [3]. The tumor occurs most commonly in young adults and adolescents in the second and third decade of life, with an age range between the ages of 10 to 30 [1]. However, cases have been previously reported outside this range. Clinical presentation is characterized by pain, swelling, and edema; most commonly noted in the proximal metaphysis of the tibia [1]. The proximal femur is also commonly affected [3]. Rarer locations include the fibula, scapula, vertebrae and ribs [3]. Occasionally clinical presentation may be absent and 


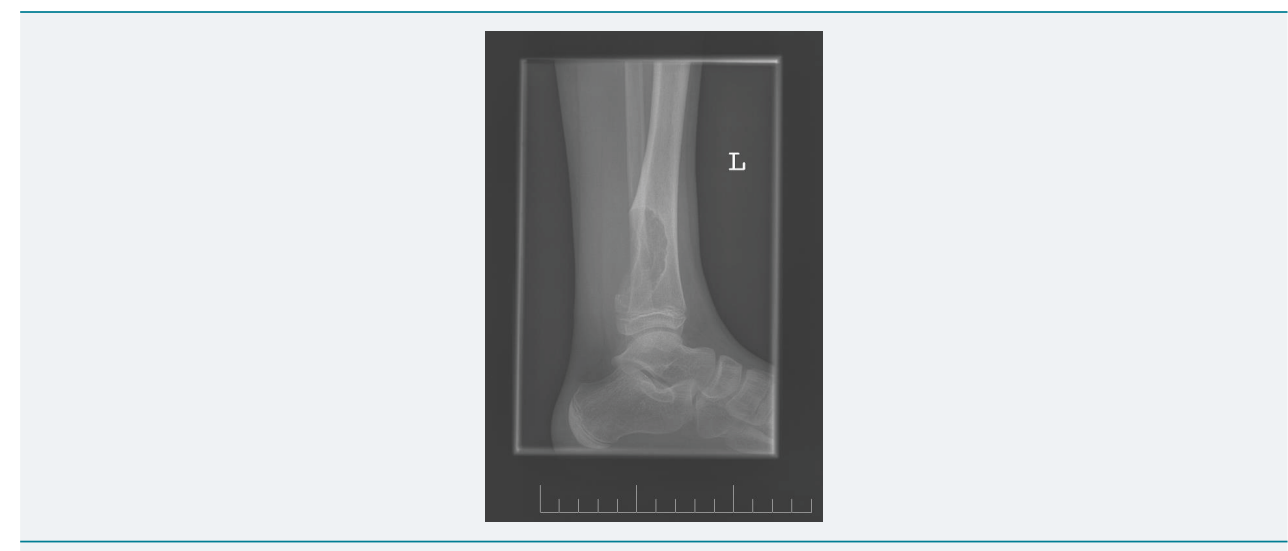

Figure 1: Initial lateral X-ray of the left ankle.

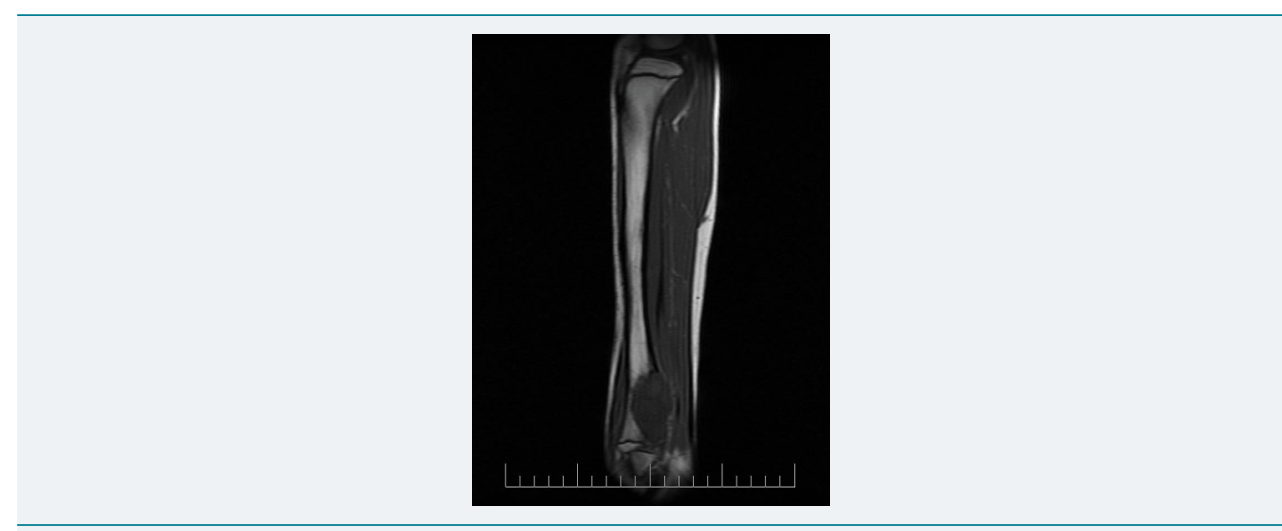

Figure 2: MRI sagittal T1 of the left tibia.

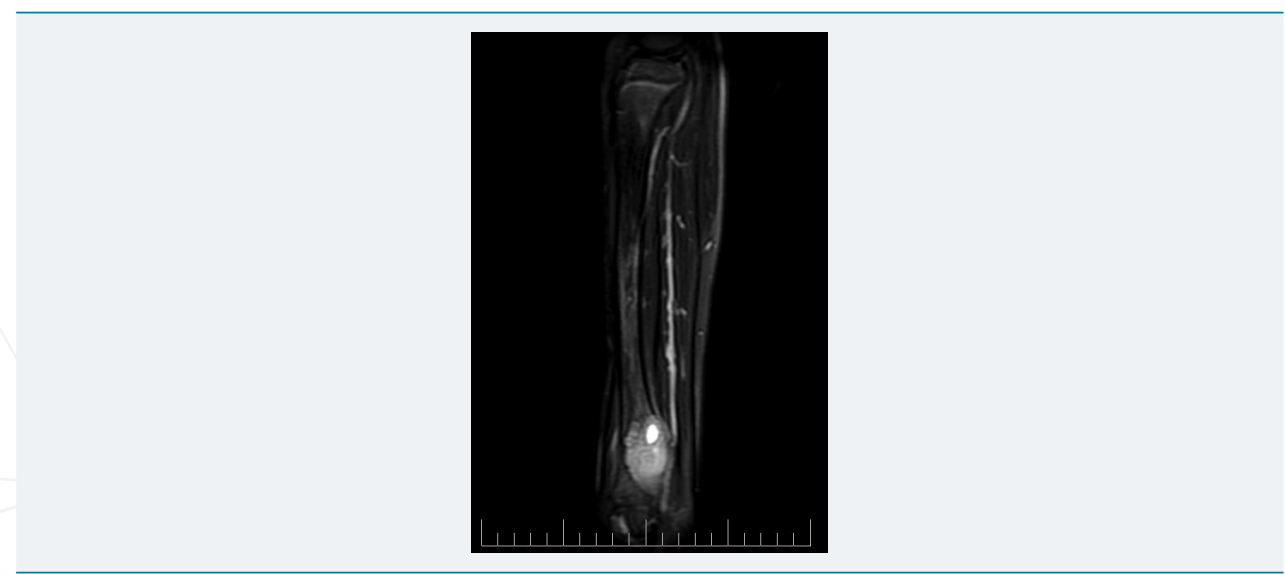

Figure 3: MRI sagittal T2 fast spin echo of the left tibia.

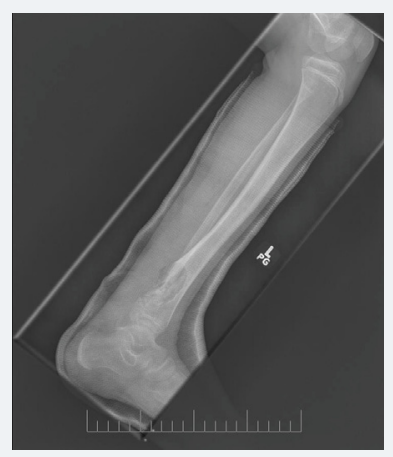

Figure 4: Post op lateral X-ray of the left tibia/fibula. 


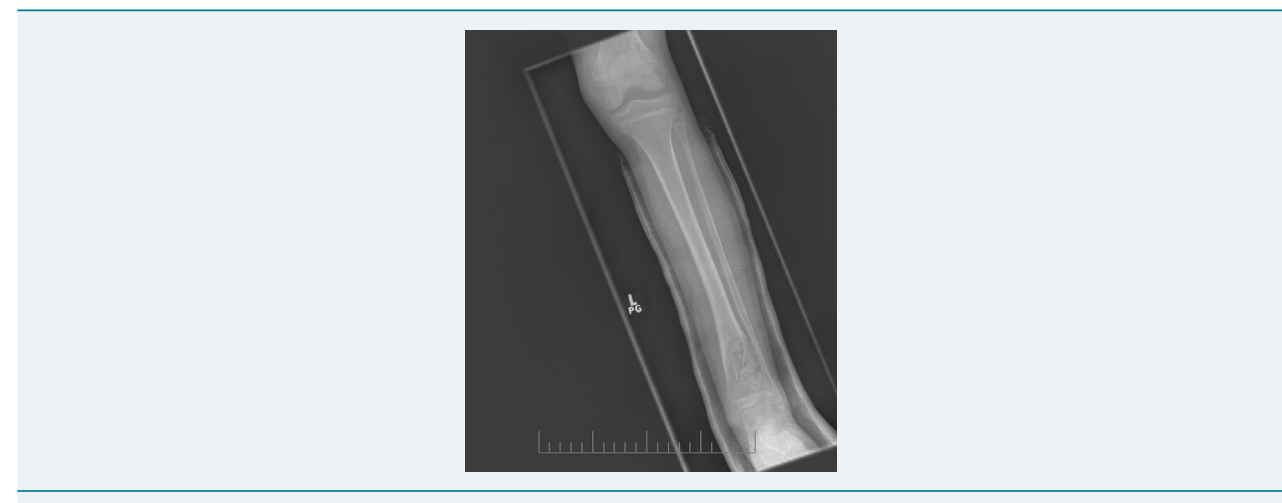

Figure 5: Post op AP X-ray of the left tibia/fibula.

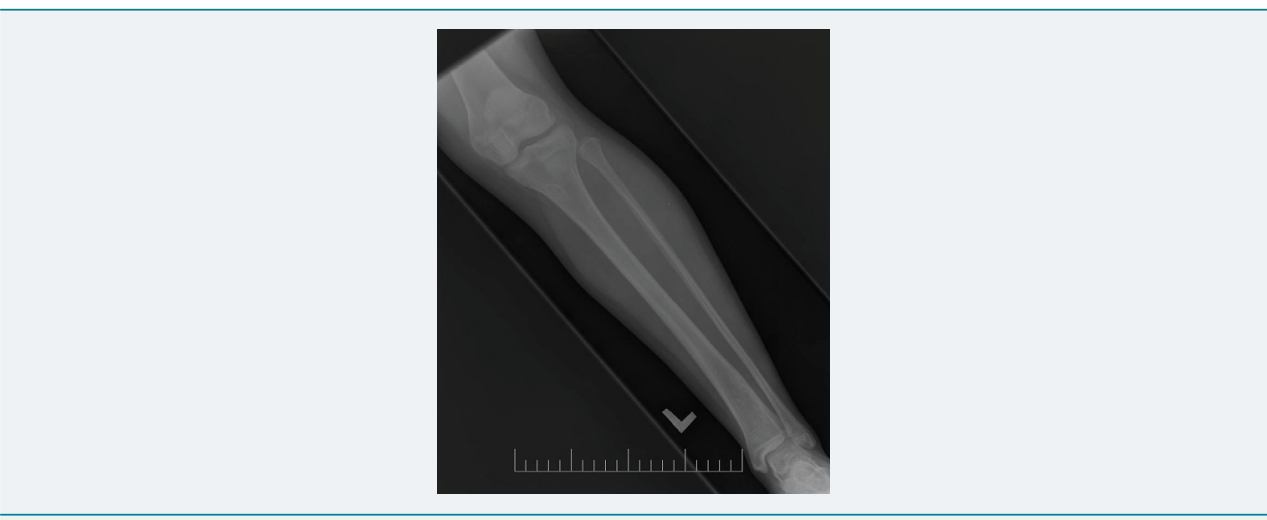

Figure 6: 5 year follow up AP X-ray of the left tibia/fibula.

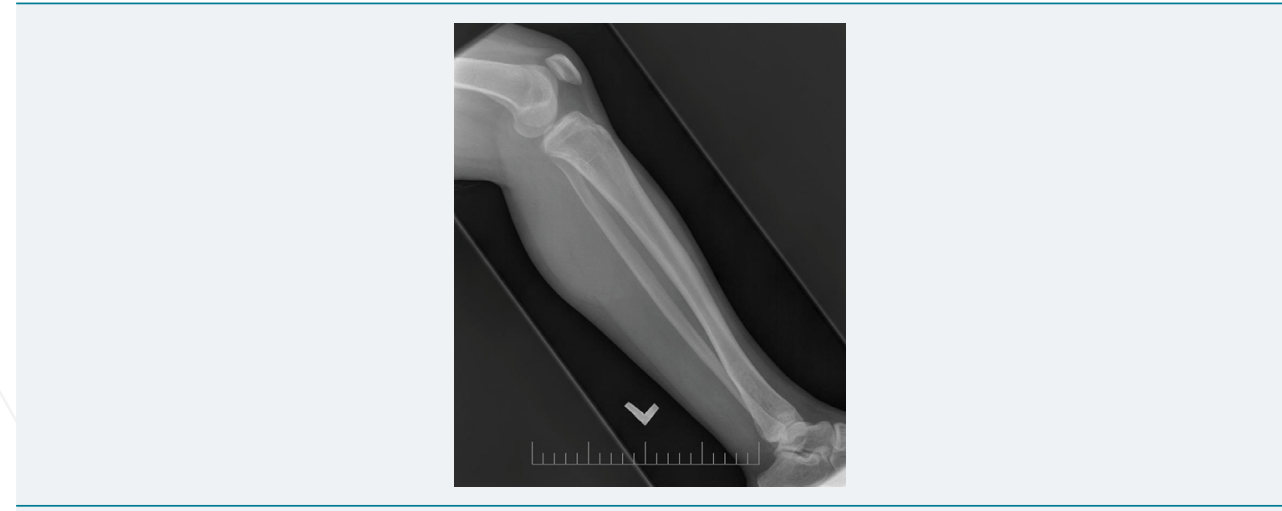

Figure 7: 5 year follow up lateral X-ray of the left tibia/fibula.

the tumor may be discovered incidentally during radiography [1]. Tumors located in the distal extremities such as the hands or feet may only present clinically with signs of painless swelling [5]. Males have a higher incidence, with no other demographic bias [3].

Imaging is crucial to the diagnosis of this tumor. On X-ray the tumor appears as a space occupying radiolucent osteolytic lesion with sharp and well-defined margins (Figure 1). The margins are noted to be sclerotic (Figure 8) the tumor has no soft tissue component (Figure 9). There is no matrix calcification or visible periosteal reaction (Figure 10).

MRI demonstrates low signal intensity on T1 weighted images (Figure 2, Figure 11 and Figure 12). T2 weighted images demonstrate high signal intensity (Figure 3, Figure 13). Post gadolinium contrast T1 weighted sagittal view demonstrates diffuse heterogenous enhancement (Figure 14). Post gadolinium contrast T1 weighted axial view demonstrates peripheral nodular enhancement (Figure 15). 


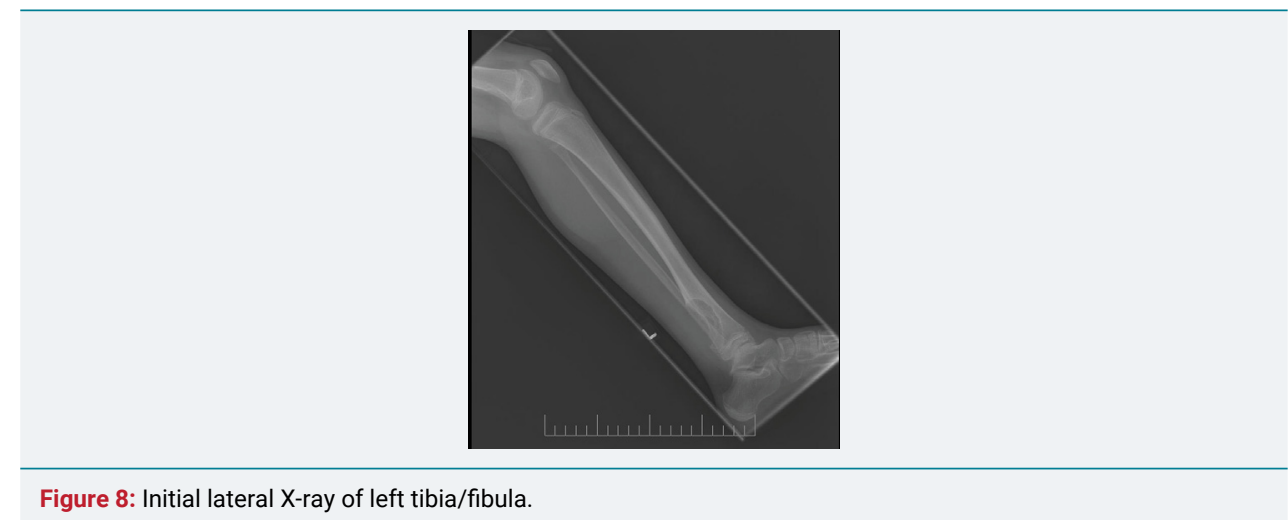

Figure 8: Initial lateral X-ray of left tibia/fibula

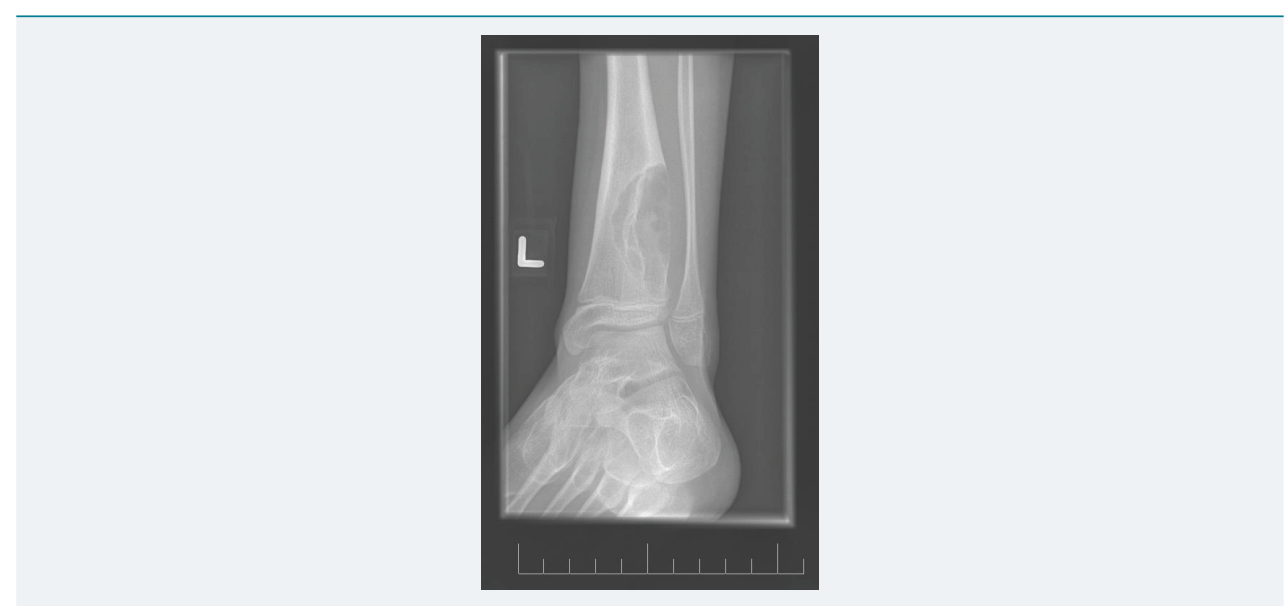

Figure 9: Initial Oblique X-ray of the left ankle.

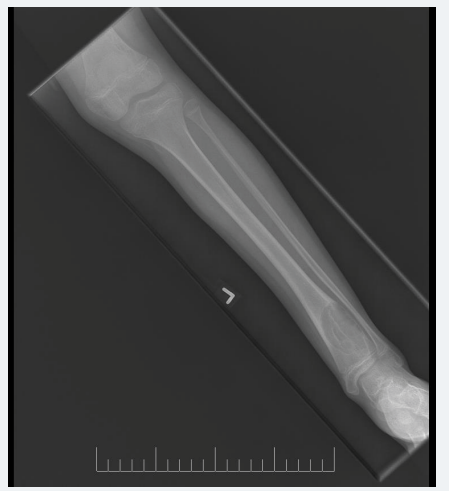

Figure 10: Initial AP X-ray of the left tibia/fibula.

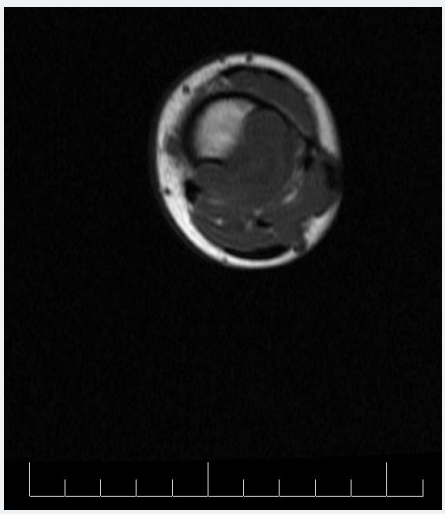

Figure 11: MRI axial T1 of the left tibia. 


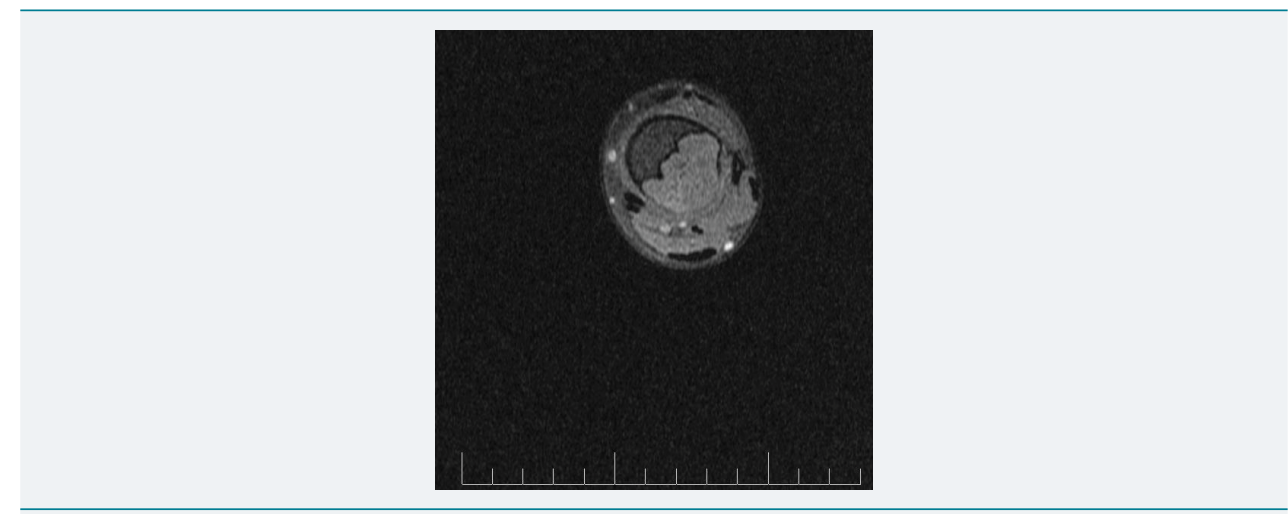

Figure 12: MRI axial T1 fat sat pre-gadolinium of the left tibia.

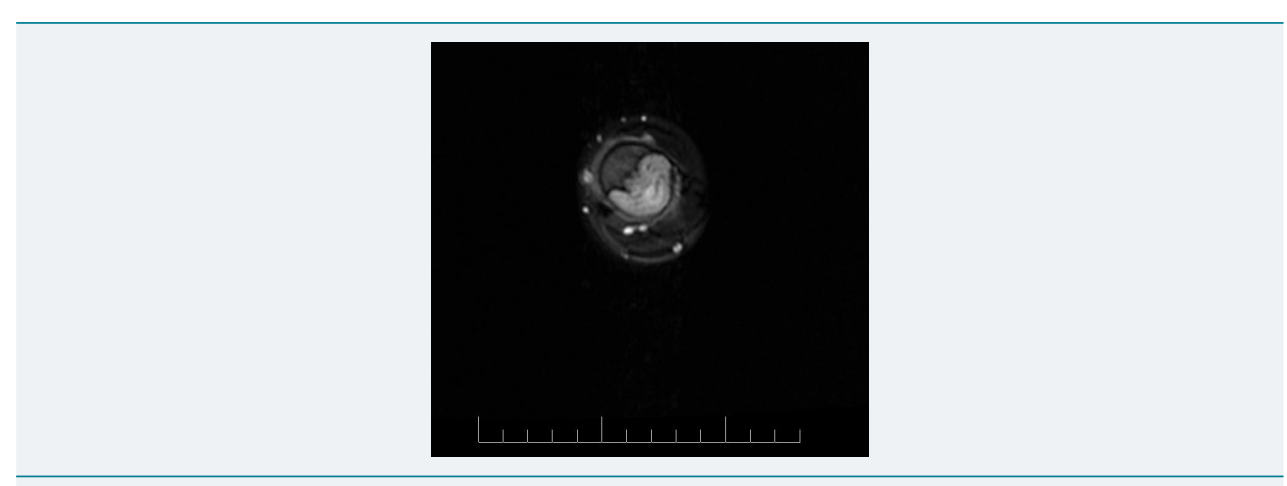

Figure 13: MRI axial T2 of the left tibia.

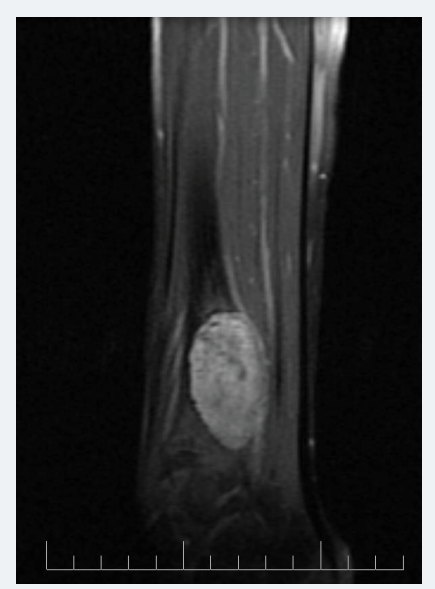

Figure 14: MRI sagittal T1 fat sat post gadolinium of the left tibia

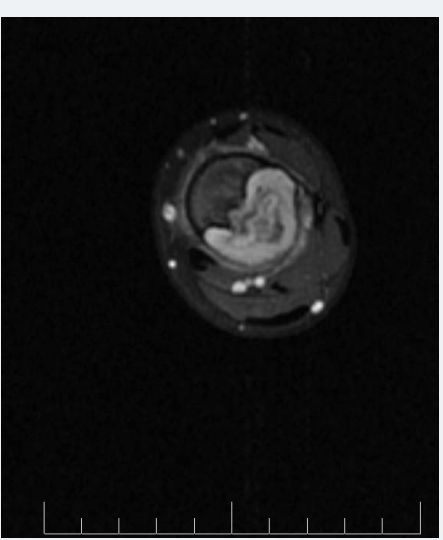

Figure 15: MRI axial T1 fat sat post gadolinium of the left tibia 
Treatment consists of curettage or en-bloc resection [6]. Curettage alone has a higher recurrence rate compared to curettage combined with bone grafting [6]. Recurrence rates as noted by Gherlinzoni F et al, were noted to be as high as $80 \%$ with curettage alone and dropping to $7 \%$ when combined with bone grafting [7]. Long bone lesions are preferably treated with either en bloc or curettage combined with bone grafting as in our case [8].

\section{CONCLUSION}

Chondromyxoid fibroma represents less than $0.5 \%$ of all bone tumors making it significantly rare as well as the rarest cartilaginous bone tumor. Common locations of occurrence include the metaphysial region of the proximal tibia and distal femur. We described a case of a 10-year-old female affected by a CMF of the left lower tibia which was treated with curettage and bone grafting. X-ray demonstrates a space occupying radiolucent osteolytic lesion with sharp and well defined sclerotic margins. MRI of the tumor revealed low signal intensity on T2 weighted images. T2 weighted images demonstrate high signal intensity. Post gadolinium contrast T1 weighted images demonstrated heterogenous enhancement. Follow up X-ray after 5 years demonstrates no tumor reoccurrence (Figure 14, Figure 15).

\section{REFERENCES}

1. Bagewadi RM, Nerune SM, Hippargi SB. Chondromyxoid Fibroma of Radius: A Case Report. J Clin Diagn Res. 2016; 10. Ref.: https://goo.gl/gQEtz8

2. Soni R, Kapoor C, Shah M, Turakhiya J, Golwala P. Chondromyxoid Fibroma: A Rare Case Report and Review of Literature. Cureus. 2016; 8: 803. Ref.: https://goo.gl/FxiLGT

3. Chowdary PB, Patil MD, Govindarajan AK. Chondromyxoid Fibroma: An Unusual Tumour at an Atypical Location. J Clin Diagn Res. 2015; 9: 4-5. Ref.: https://goo.gl/U9u3BV

4. Giudici MA, Moser RP Jr, Kransdorf MJ. Cartilaginous bone tumors. Radiol Clin North Am. 1993; 31: 237-259. Ref.: https://goo.gl/jfsYKS

5. Sutton D. 7th ed. Edinburgh: Churchill Livingstone. Textbook of radiology and imaging. 2003.

6. Dulani R, Dwidmuthe SC, Shrivastava S, Singh P, Gupta S. Huge chondromyxoid fibroma of proximal third tibia masquerading as an aneurysmal bone cyst: A rare case report. South Asian $\mathrm{J}$ Cancer. 2013; 2: 13. Ref.: https://goo.gl/Ui6J9e

7. Gherlinzoni F, Rock M, Picci P. Chondromyxoid fibroma. The experience at the Instituto Ortopedico Rizzoli. J Bone Joint Surg Am. 1983; 65: 198-204. Ref.: https://goo.gl/Yvkfut

8. Nagaraj S. Chondromyxoid fibroma of calcaneum. International Journal of Biomedical Research. 2014; 5: 3926-28. 